\title{
Prediction of 'highly skin smear positive' cases among MB leprosy patients using clinical parameters
}

\author{
JOSEPH W. LEMASTER*, TIN SHWE**, C. RUTH \\ BUTLIN $* * *$ \& PAUL W. ROCHE*** \\ * Department of Family Medicine, University of Washington, P.O. \\ 354696, Seattle, Washington (WA) 98195 \\ **Leprosy Control Division, HMG, Nepal \\ *** Mycobacterial Research Laboratory, Anandaban Leprosy \\ Hospital, PO 151, Kathmandu, Nepal
}

\section{Accepted for publication 18 October 2000}

\begin{abstract}
Summary Although 'highly skin smear positive' MB leprosy cases are known to be at high risk of relapse after release from treatment, and have been recommended to receive 'prolonged duration' MDT, government field-based control programmes without skin smear facilities have no simple alternative method to detect such cases. This study reports a significant prevalence of 'highly smear positive' cases amongst 2374 new multibacillary cases recently surveyed by skin smears in Nepal, and retrospectively analyses 555 newly detected, previously untreated BL and LL cases to identify clinical and laboratory parameters that may be associated with a 'highly positive skin smear'. While some parameters showed high sensitivity in predicting 'highly positive smear' status, none showed both high sensitivity and high specificity simultaneously.
\end{abstract}

\section{Introduction}

Recently the WHO Expert Committee on Leprosy has concluded that the length of fixed duration multi-drug therapy (MDT) for multibacillary (MB) patients could be shortened to 12 months, without greatly increasing the risk of relapse for the majority of such patients. ${ }^{1}$ There has been much debate over this topic in the last year among national control programmes, who have the responsibility for implementing this decision in their own countries. The risk of relapse among MB leprosy cases who have received the standard 24 months of MDT has been found to be as low as $0.77 \%^{2}$ and as yet, there is no evidence that shorter courses of therapy will increase the risk of relapse. ${ }^{3,4}$ However, a high rate of relapse $(39 \%)$ has been reported among MB patients with 'highly positive slit skin smears' (bacterial index $(\mathrm{BI}) \geq 4.0+$ ) treated with 24 months of standard MDT. ${ }^{5}$ Pattyn in Zaire and others have suggested that the

Correspondence: J. W. LeMaster (e-mail: lemaster@gateman.com) 
higher the BI, or the shorter the duration of therapy, the higher the risk of relapse, ${ }^{6,7}$ and many programmes wish to treat highly smear positive (HSP) patients with at least 24 months MDT.

In Nepal, a diagnosis of MB leprosy is based on clinical findings, not on slit skin smear results. Newly detected cases in Nepal who have disease in more than two body areas are classified as multibacillary, and until recently all were given 24 months fixed duration MDT. Despite the national policy to treat HSP cases with 24 months of MDT, there is no skin smear referral system available throughout in Nepal. Government peripheral health posts must continue to classify cases on the basis of clinical findings, and from 1999 have treated all MB cases with the 12-month MDT regimen.

In 1999, slit skin smears were taken from 2374 new previously untreated MB cases representing about $10 \%$ of the new cases detected during a national leprosy elimination campaign. At the time of skin smear, all cases had 1-6 months of MDT treatment. Of these patients, $18.2 \%$ (433) were found to be skin smear positive, and 8.2\% (193) had either an average $\mathrm{BI}>3 \cdot 0$, or at least one lesion with $\mathrm{BI} \geq 4 \cdot 0$ (Nepal Leprosy NGO Network meeting minutes, August 1999). Thus, there is a substantial proportion of HSP MB cases in field programmes in Nepal, but without skin smear services there is no way to identify these high-risk cases in the field-based programme.

This study attempts to identify whether a particular clinical feature or combination of features can be used in a field setting to identify cases with 'highly positive' skin smears.

\section{Materials and methods}

We used data collected retrospectively from a previously described sample of MB cases. ${ }^{8}$ Data from 649 previously untreated MB patients diagnosed on clinical and bacteriological grounds, who presented to Anandaban Leprosy Hospital between 1989 and 1997 were used. Ninety-four cases were excluded from the analysis because an initial skin smear had not been performed. The remaining 555 cases provided the data for this analysis. Clinical, bacteriological and serological information collected at the time of first presentation and diagnosis was reviewed. Slit skin smears were taken from all 555 cases, and the mean BI was calculated from smears taken from a minimum of four sites including a clinical lesion. The Disability Index (DI) according to the WHO classification system was recorded for 460 patients. In addition, the number of body areas with disease was recorded for 550 cases, based on the number of areas in which there were either skin patches or enlarged nerves. The maximum number of body areas possibly affected was nine, consisting of four for the trunk (divided anterior and posterior sagittally), four for the limbs and one for the head. The total number of skin patches (for 364 patients), enlarged, palpable nerve trunks (for 529 patients), the presence of diffuse infiltration and of lepromatous (non-ENL) nodules (both for 555 patients) was also recorded. IgM anti-PGL-1 antibodies were measured from serum in 450 cases. We also recorded the patient's estimate of the numbers of years since the first appearance of leprosy disease: this gave a measure of the period of untreated disease before treatment started. ENL reactions at presentation were also recorded.

Differences between HSP and other cases with respect to quantitative variables were tested using the Mann-Whitney $U$-test. Unadjusted odds ratios and 95\% confidence intervals were calculated from two-way contingency tables, comparing the proportions of those with and without HSP for each clinical characteristic examined. Multiple logistic regression was used to control for confounding between the predictors, and to calculate adjusted odds ratios 
Table 1. Relationship between clinical and laboratory parameters and the prevalence of highly positive skin smears $(\mathrm{BI} \geqslant 4+)$

\begin{tabular}{|c|c|c|c|c|c|c|}
\hline Predictor & $\begin{array}{l}\text { No. HSP/total } \\
\text { positive (PPV) }\end{array}$ & $\begin{array}{l}\text { No. HSP/total } \\
\text { negative (NPV) }\end{array}$ & $\begin{array}{l}\text { Unadjsuted OR } \\
\quad(95 \% \mathrm{CI})\end{array}$ & $\begin{array}{c}\text { Adjsuted OR } \\
(95 \% \mathrm{CI})\end{array}$ & $\begin{array}{c}\text { Sensitivity } \\
(\%)\end{array}$ & $\begin{array}{c}\text { Specificity } \\
(\%)\end{array}$ \\
\hline LL classification & $137 / 211(65 \%)$ & $78 / 344(23 \%)$ & $6.3(4.1-9.6)^{* *}$ & $6.8(3.0-15.8)^{* * *}$ & 64 & 78 \\
\hline Age $>40$ years & $89 / 246(36 \%)$ & $126 / 308(41 \%)$ & $0.81(0.6-1.1)$ & $\mathrm{n} / \mathrm{a}$ & 41 & 54 \\
\hline Male sex & $167 / 417(40 \%)$ & $48 / 137(35 \%)$ & $1.2(0.8-1.8)$ & $\mathrm{n} / \mathrm{a}$ & 78 & 26 \\
\hline Body areas with disease $>5$ & $204 / 485(42 \%)$ & $11 / 65(17 \%)$ & $3.6(1.8-6.9) * * *$ & $10.1(2.3-43.8)^{* *}$ & 95 & 16 \\
\hline Enlarged nerves $>4$ & $167 / 364(46 \%)$ & $42 / 165(25 \%)$ & $2.4(1.6-3.7)^{* * *}$ & $1.0(0.4-2.5)$ & 80 & 39 \\
\hline Skin patches $>5$ & $48 / 195(25 \%)$ & $88 / 169(52 \%)$ & $0.3(0.19-0.47)^{* * *}$ & $0.7(0.3-1.5)$ & 35 & 35 \\
\hline Skin infiltrate & $193 / 364(53 \%)$ & $22 / 191(12 \%)$ & $8.2(4.9-13.9)^{* * *}$ & $3.7(1.5-9.0)^{* *}$ & 90 & 50 \\
\hline Skin nodules & $68 / 99(69 \%)$ & $147 / 456(32 \%)$ & $4.6(2.8-7.5)^{* * *}$ & $1.9(0.8-4.9)$ & 32 & 91 \\
\hline anti-PGL-1Ab sero-positive & $152 / 319(48 \%)$ & $29 / 131(22 \%)$ & $3.2(2.0-5.1)^{* * *}$ & $1.7(0.7-4.1)$ & 84 & 38 \\
\hline ENL reaction (at presentation) & $18 / 33(24 \%)$ & $197 / 522(38 \%)$ & $2.0(1.0-4.0)^{*}$ & $1.3(0.3-5.1)$ & 31 & 88 \\
\hline Established disability & $33 / 148(22 \%)$ & $142 / 312(46 \%)$ & $0.35(0.22-0.54)^{* * *}$ & $0.4(0.2-0.9)$ & 19 & 60 \\
\hline
\end{tabular}

${ }^{*} P<0.05,{ }^{* *} P<0.01,{ }^{* * *} P<0 \cdot 001 . \mathrm{PPV}=$ positive predictive value NPV $=$ negative predictive value. 
for those cases for which all clinical and laboratory parameter data was available (291 cases). Specificity, sensitivity, and positive predictive value for a diagnosis of HSP were calculated from each predictor or two-way combination of predictors.

\section{Results}

All information was collected at the time of diagnosis. Of the cases in the sample, $344(62 \%)$ were classified as borderline lepromatous (BL) on clinical grounds at first presentation and $211(38 \%)$ as polar lepromatous (LL). Prevalence odds ratios describing the risk relationships between clinical and laboratory parameters and HSP status are shown in Table 1. Patients with highly positive skin smears $(\geq 4+)$ had significantly more body areas with leprosy disease, more enlarged nerve trunks and higher levels of anti-PGL-1 antibodies than patients with skin smears with lower BI.

We analysed each clinical feature to assess the strength of association with classification of a case as HSP, and calculated the sensitivity, specificity, and positive and negative predictive values for a diagnosis of an HSP skin smear. An LL classification, the presence of skin infiltrate, the presence of lepromatous nodules, the presence of ENL, anti-PGL-1 antibody seropositivity, more than five body areas affected by leprosy, and more than four nerves enlarged, all predicted significantly increased risk of a case being HSP. The presence of six or more skin patches or a DI $>1$ both predicted a decreased risk for HSP. Neither age greater than 40 years nor male sex was predictive of HSP.

Because a number of the clinical features shown in Table 1 appeared to be strongly predictive of HSP, we used a logistic regression model to control for potential confounding amongst the most strongly predictive features. These 'adjusted odds ratios' are also shown in Table 1. In this model, having more than five body areas with disease was the most sensitive predictor, whereas the presence of lepromatous nodules was the most specific. No single predictor simultaneously showed a sensitivity and specificity in excess of $64 \%$.

The three clinical features, LL classification, more than five body areas with disease and skin infiltration, were combined to measure the effect on HSP prediction. In each case, if either one or the other of the two clinical features was present, HSP was predicted. Despite odds ratios that were generally stronger than for either clinical feature on its own, the sensitivity of these combinations was high, up to $98 \%$, but specificity and positive predictive values were low.

Table 2. Effect of combinations of clinical characteristics in predicting high skin smear positivity

\begin{tabular}{llccc}
\hline Symptoms & Odds ratio $(95 \%$ CI) & $\begin{array}{c}\text { Sensitivity } \\
(\%)\end{array}$ & $\begin{array}{c}\text { Specificity } \\
(\%)\end{array}$ & $\begin{array}{c}\text { Positive predictive } \\
\text { value }(\%)\end{array}$ \\
\hline More than five body areas or skin infiltration & $7.7 * * *(2.2-25.9)$ & 98 & 9.8 & 41 \\
More than five body areas or LL class & $1.4(0.6-3.2)$ & 96 & 6.1 & 39 \\
Skin infiltration or LL class & $1.7(0.7-3.9)$ & 96 & 5.9 & 39 \\
Any feature positive & $3.6(0.8-16.4)$ & 99 & 3.2 & 39 \\
All features positive & $0.9(0.6-1.3)$ & 29 & 68 & 37 \\
\hline
\end{tabular}

$* * * P<0.001$. 
Combination of all three clinical features in Table 2 did not yield any additional advantage. As one might expect, if any of the three features was accepted as predictive of HSP status, sensitivity approached $100 \%$, while specificity fell towards $0 \%$. The opposite was true if we required the simultaneous presence of all three features to predict HSP status: increases in specificity were at the cost of decreases in sensitivity.

\section{Discussion}

The practical utility of the clinical features examined here in identifying HSP cases is marginal. No single clinical feature simultaneously showed both sensitivity and specificity in excess of $65 \%$. This was also true of all the combinations of clinical features examined. However, some of the single features and some of the combinations of features did show very high sensitivity. Control programmes must examine their priorities in order to interpret and use these results. If the highest priority is given to identifying virtually all HSP cases, and the unnecessary treatment of up to $50 \%$ of those who are not, in fact, 'highly smear positive' is acceptable from a logistical and cost basis, a programme might choose to use one or more of the most sensitive clinical features identified here as a screening tool. However, the usual reality for control programmes is that they must conserve scarce resources, making sure that a majority of those predicted to be HSP are so before providing additional testing, treatment or follow-up for such cases. From that perspective, none of these clinical features or combinations of features is very acceptable. It must also be remembered that the positive predictive value for a clinical feature will depend on the prevalence of that feature. ${ }^{9}$ In field populations in Nepal, where only $8 \%$ of cases classified as MB are HSP, the positive predictive value of these clinical features will be much poorer than in this study.

One needs to look carefully at what action could be taken even if it were possible to identify HSP cases. The Institute Marchoux group recommended that MB cases with an initial average $\mathrm{BI} \geq 4.0$ receive at least 4 years of standard WHO MDT; ${ }^{5}$ however, to our knowledge the efficacy of this treatment has not yet been tested. As yet, no clear international guidelines have been developed regarding the optimal duration of treatment for such cases. Further studies should be undertaken to clarify this matter urgently.

The cost of treating either all or a targeted sub-population of MB cases with MDT for a prolonged period or carefully following the same sort of patients for a decade seems high initially, but may not be excessive. If relapse among the 'highly smear positive' cases proves as frequent as was found by the Marchoux group (39\%), and HSP cases are as common as the Nepal survey estimated, $31 \mathrm{MB}$ cases would need to be treated with 'prolonged' MDT or followed up for 8-10 years to prevent one 'highly smear positive' case from relapsing. This needs to be weighed against the lower costs of re-treatment of the relapses with a second course of MDT, assuming that there is no development of drug resistant disease nor the development of additional disability in relapse cases. Until a large cohort of MB cases, who have received only 12 months of MB MDT, have been followed after release from treatment for 7-10 years, it will be impossible to assess the scale of the relapse rate among HSP patients. Whatever decisions are ultimately made regarding the management of HSP cases, control programmes must weigh the social and medical consequences for individual cases who may relapse against the significant logistical and financial obstacles that will have to be overcome by the programme to ensure that all such cases are treated adequately. 


\section{Acknowledgements}

This study was supported by the Leprosy Mission International and the Nepal Leprosy NGO Network. We wish to thank Ms Ros Walley for her helpful comments and advice on the statistics.

\section{References}

${ }^{1}$ WHO Expert Committee on Leprosy, Seventh Report. WHO Technical Report Series, No. 874. World Health Organisation, Geneva, 1998.

${ }^{2}$ Leprosy Unit, Division of Control of Tropical Diseases. Risk of relapse in leprosy. WHO/CTD/LEP/94.1. World Health Organisation, Geneva, 1994.

${ }^{3}$ Ganapati R, Revankar CR, Pai W, Girija D. Fixed duration multidrug therapy in leprosy—a preliminary follow-up report. $15^{\text {th }}$ International Leprosy Congress, Beijing (abstract $\mathrm{CH} 37$ ).

${ }^{4} \mathrm{Ji}$ B. Why multidrug therapy for multibacillary leprosy can be shortened to 12 months. Lepr Rev, 1998; 69: 106109.

5 Jamet P, Ji B, and the Marchoux Chemotherapy Study Group. Relapse after long-term follow-up of multibacillary patients treated by WHO multidrug regimen. Int J Lepr 1995; 63: 195-201.

6 Marchoux Chemotherapy Study Group. Relapses in multibacillary leprosy patients after stopping treatment with rifampin-containing combined regimens. Int J Lepr 1992; 60: 525-535.

${ }^{7}$ Waters MFR. Relapse following various types of multidrug therapy in multibacillary leprosy. Lepr Rev 1995; 66: $1-9$.

${ }^{8}$ Manandhar R, LeMaster J, Roche P. Risk factors for erythema nodosum leprosum. Int J Lepr 1999; 67: 270-278.

${ }^{9}$ Henneken CH, Buring JE. Epidemiology in medicine. Little, Brown and Co., Boston, USA, 1987, pp 337-339. 